\title{
Laser Treatment of Darier Disease: Report of Two Cases and Systematic Review of the Literature
}

\author{
Kathleen F O'Brien ${ }^{1}$, Michelle A Fricke ${ }^{2}$, Rhett A Kent ${ }^{3}$, Cynthia Marie Carver DeKlotz ${ }^{1,2^{*}}$ \\ ${ }^{1}$ Georgetown University School of Medicine, Washington, DC, USA \\ ${ }^{2}$ MedStar Washington Hospital Center, Chevy Chase, MD, USA \\ ${ }^{3}$ Forefront Dermatology, Arlington, VA, USA
}

\author{
*Correspondence to \\ Cynthia Marie Carver DeKlotz \\ M.D; \\ Address: 5530 Wisconsin Ave, \\ Suite 730, Chevy Chase, MD \\ Tel: 301-951-2400; \\ Fax: 301-951-2401; \\ Email: cmc49@georgetown.edu \\ Published online October 3 \\ 2020
}

\begin{abstract}
Introduction: Darier disease (DD) is a chronic disease with high morbidity and limited treatment options. Laser efficacy in the treatment of DD remains understudied.

Methods: A literature search conducted between 07/21/2017 and 05/05/2018 identified all original cases of DD treated with laser therapy.

Results: Outcomes from 24 patients were reviewed, 22 patients were identified in the literature and 2 cases are included from our institution. Five types of lasers were identified $\left[\mathrm{CO}_{2}\right.$ laser, Er:YAG laser, pulse dye laser [PDL], diode laser, erbium-doped fiber laser], with $\mathrm{CO}_{2}(46 \%)$ and PDL (42\%) being the most common. Seventy-nine percent of patients were treated with more than one procedure (average, 3 procedures). The estimated body surface treated with each session ranged from 5\%-50\%. The most common adverse events came from $\mathrm{CO}_{2}$ and Er:YAG lasers and included dyspigmentation, pain, and post-laser erythema and edema. The time to response (mode, 1 month) was only reported in half of the studies.

Conclusion: Laser treatments appear to be a promising alternative to standardized therapies in DD. Keywords: Laser; Treatment; Darier disease; Keratosis follicularis.
\end{abstract}

\section{Introduction}

Darier disease (DD), also known as keratosis follicularis, is a rare autosomal dominant disorder due to ATP2A2 gene mutations that encode SERCA2 protein, a calcium ATP-ase. ${ }^{1}$ Cutaneous findings associated with the disease can appear as early as age four but typically present in the second or third decades of life. The SERCA2 protein dysfunction leads to calcium dysregulation in epidermal keratinocytes, resulting in a rash. ${ }^{1}$ The rash is characterized by dark crusty thickened and hyperkeratotic patches on the skin. The rash is exacerbated by trauma, heat, sweating, and ultraviolet light. ${ }^{2}$

DD is a chronic disease with high morbidity and limited treatment options. The goals of treatment include reduction of odor, infections, and discomfort, as well as the improved cosmetic outcome. Current therapies include topical and oral retinoids, steroids, and calcineurin inhibitors. ${ }^{3}$ Surgical excision, dermabrasion, or laser therapy have been considered for recalcitrant disease. $^{2}$

Although retinoids remain the standard treatment for DD, lasers are gaining interest as a therapeutic alternative. Here we discuss all published studies evaluating the efficacy of the lasers in the treatment of DD and include two never before reported cases from our institution.

\section{Methods}

Search Strategy

We conducted an electronic search of PubMed, EMBASE, NLLSCA, and Cochrane Library databases for articles that evaluated the efficacy of any laser type in the treatment of DD. Our search was conducted between 07/21/2017 and $05 / 05 / 2018$. The search was performed using the following terms in combination: darier('s) disease, darier(-)white disease(s), acantholytic dyskeratotic epidermal nevi/nevus, acrokeratosis verruciformis (of hopf), keratosis follicularis, laser(s), continuous wave laser(s), pulsed laser(s), q-switched laser(s), and laser(s). We also manually searched the reference list of relevant articles for additional review.

\section{Inclusion Criteria}

The pre-defined inclusion criteria included all sources, written in the English language, presenting any original cases $(\mathrm{N}>0)$ of DD entities being treated with any laser.

\section{Data Abstraction}

The PRISMA checklist, as detailed by Moher et al in 2009, was used as a guide for completing the necessary steps of this systemic review. ${ }^{4}$ All articles were then evaluated to determine the efficacy of the laser types

Please cite this article as follows: O'Brien KF, Fricke MA, Kent RA, DeKlotz CMC. Laser treatment of Darier disease: report of two cases and systematic review of the literature. J Lasers Med Sci. 2020;11(4):395-404. doi:10.34172/jlms.2020.63. 
used in the treatment of DD. Two reviewers, R.A.K. and K.F.O, independently performed the search with the help of academic librarians in a medical library. Three reviewers, R.A.K, M.A.F, and K.F.O, independently assessed abstract eligibility for inclusion using the preestablished criteria. Once complete, discordant abstracts were assigned to inclusion/exclusion groups based on further review and ultimately, agreement by reviewers. Selected abstracts were then reviewed in detail by two independent reviewers, K.F.O and M.A.F, who completed data extraction using a standardized data collection form. A fourth reviewer, C.M.C.D, independently checked for the accuracy of details and their application. The primary authors were contacted for unpublished data if necessary.

\section{Results}

Case Presentations

We report 2 new cases of refractory DD that improved with fractional $\mathrm{CO}_{2}$ laser treatments.

Patient $A$ is a 32-year-old Caucasian female with a 30 -year history of genetically confirmed DD, who failed treatment with topical steroids, topical retinoids, and oral isotretinoin. Patient B is a 43 -year-old Caucasian female with a 39-year history of genetically confirmed DD, who failed oral and topical steroids, topical tacrolimus, and oral acitretin. Both patients had involvement of $>80 \%$ body surface area (BSA) and were Fitzpatrick skin type II. Both patients' courses were complicated by recurrent infections requiring hospitalization, intractable pain, and pruritus.

\section{Treatment}

The UltraPulse Lumenis fractionated CO2 laser was used for both Patient A and Patient B, and Patient B also underwent a single procedure with the SSI Laser Engineering Ultra MD 60 with a fractionated handpiece. The UltraPulse Lumenis fractionated $\mathrm{CO}_{2}$ laser was set to $10600 \mathrm{~nm} \mathrm{CO}_{2}$ ultra pulse setting. Specifically, the ultrapulse setting was $600-625 \mathrm{~Hz}$, with an exposure time of 10 milliseconds (ms), and repeat delays ranging from 0.3 to 1 second. The determination of millijoules $(\mathrm{mJ})$, ranging from 10-100 $\mathrm{mJ}$, was based on the approximated thickness and location of the skin (higher $\mathrm{mJ}$ for thicker areas on the trunk and extremities). The ALMA PIX1 handpiece was used with $5 \%$ surface area coverage. Preoperatively, the sites were cleaned with Chloraprep and allowed to dry completely. After each procedure, laser-treated areas were covered with white petroleum and ice.

Patient $A$ underwent a total of 3 fractional $\mathrm{CO}_{2}$ laser treatments spaced $4-6$ weeks apart. The abdomen $(<10 \%$ TBSA) was treated in the first procedure. The treated area was divided into thirds and treated differently. One area was treated with a single pass using the ultrapulse setting at $10 \mathrm{~ms}$, the second area with a single pass using the continuous wavelength setting $(40 \mathrm{~W}, 100 \mathrm{~ms}$, with 1-second repeat delays) and the third area with a single pass of both laser settings (specifically, the ultrapulse setting at $10 \mathrm{~ms}$ followed by the continuous wavelength setting at $100 \mathrm{~ms}$ ). The best results objectively and subjectively were noted in the area treated with only one pass using the ultrapulse setting. Therefore, the ultrapulse setting at $10 \mathrm{~ms}$ was used for all future treatments. During the following two procedures, in addition to the abdomen, the face, neck, chest bilateral arms and legs (TBSA of 40\%$50 \%)$ were also treated.

Patient $B$ underwent a total of 12 fractional $\mathrm{CO}_{2}$ laser treatments spaced 1-5 months (mode, 2 months) apart, with varying body areas being treated on each visit. Each procedure involved a single pass of the laser. The TBSA targeted each visit was between 10\%-40\% (mode, 20\% TBSA). With the exception of the first procedure, the thick hyperkeratotic areas on Patient B's body, specifically the abdomen, chest, back and extremities, were treated with topical Kenalog $40 \mathrm{mg} / \mathrm{mL}$ at the conclusion of each laser procedure. To the best of our knowledge, this is the first report of using laser-assisted corticosteroid drug delivery in the treatment of DD.

\section{Outcome}

Patient A improved noticeably after 2 procedures. She demonstrated immediate softening and improved epidermal texture in the operating room and experienced post-laser erythema. Pruritus improved significantly, with $60 \%$ reduction overall and near resolution in some treated areas; the patient commented on a follow-up visit that she "forgot she used to itch constantly." She was found to have approximately $30 \%-50 \%$ aesthetic improvement.

Patient $B$ experienced noticeable improvement after the first procedure (Figure 1). She demonstrated immediate softening and improved epidermal texture in the operating room, most noticeably on areas that were most severe clinically and symptomatically. Subjectively she noted decreased flares and decreased pain and pruritus in treated areas. After an area of 35\%-40\% TBSA was treated (procedure \#2), she subsequently experienced a substantial systemic DD flare. All subsequent procedures

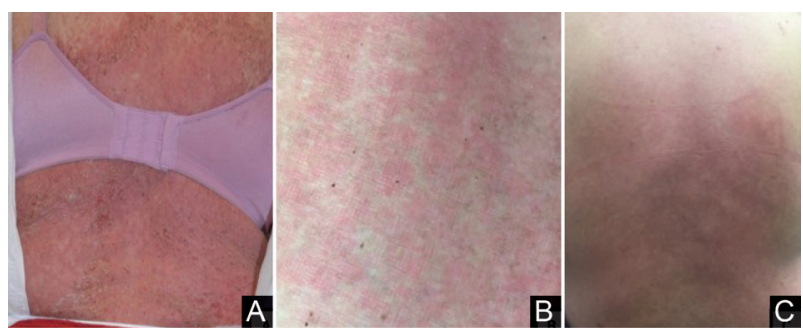

Figure 1. Patient B's Initial Response to the UltraPulse Lumenis Fractionated $\mathrm{CO}_{2}$ Laser Treatment. (A) Pre-treatment: Patient B's back at her initial presentation. (B) Post laser erythema and skin softening were noted immediately after the first fractional CO2 laser treatment. (C) Significant improvement in skin texture and color was noted eight days after the first fractional $\mathrm{CO} 2$ laser procedure. 
were therefore minimized to a surface area of less than $25 \%$, which resulted only in minimal post-laser erythema and no additional laser-induced systemic DD flares. Patient $B$ continues to have widespread disease, but the sites treated with the $\mathrm{CO}_{2}$ laser demonstrate significantly fewer flares, itching, and discomfort. Currently, she demonstrates approximately $75 \%$ clearance and approximately $75 \%$ aesthetic improvement. Patient $B$ continues to undergo treatment at this time.

\section{Review of Literature}

Patient Population

Twelve studies met the inclusion criteria (Figure 2). Eleven case reports and 1 case series were included. The study specific characteristics of each article and patient are listed in Table 1 with additional details and specifications listed in Table S1 (see Supplementary file 1). ${ }^{5-15}$ With the addition of our two cases, a total of 24 patients met the inclusion criteria (Table 1). Of the Fitzpatrick skin types reported, or estimated by reviewer K.F.O based on clinical images, the most common was type $2(9 / 13 ; 69 \%)$. Twelve individuals (50\%) had biopsy, histological or genetically confirmed DD. Common therapies that failed prior to laser treatment included topical retinoids $(n=11)$, topical corticosteroids $(n=10)$ and oral retinoids $(n=15)$. The most common symptoms necessitating laser treatment were appearance $(n=5)$, infection $(n=4)$, pain $(n=4)$, and irritation $(n=10)$.

\section{Treatment Characteristics}

Five types of lasers were identified in our review, which included $\mathrm{CO}_{2}(\mathrm{n}=11)$, Erbium:doped yttrium aluminum garnet (Er:YAG; $n=2)$, pulse dye laser (PDL; $n=10$ ), diode laser $(n=1)$, and 1,450-nm erbium-doped fiber laser $(n=1)$. The number of laser treatments ranged from one to greater than 20. Seventy-nine percent of patients

35 Records identified through database searching

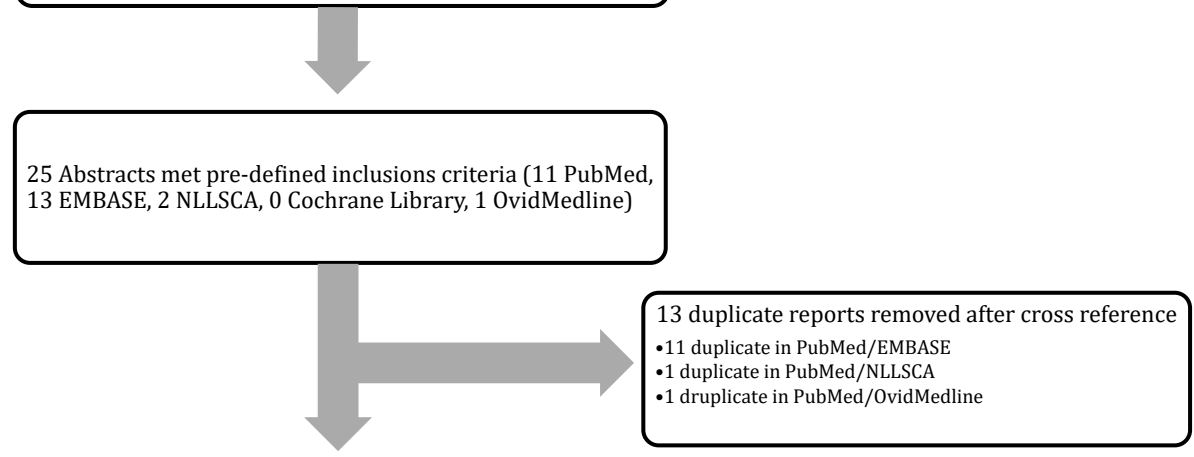

12 Origional abstracts screened after cross reference and duplicates removed

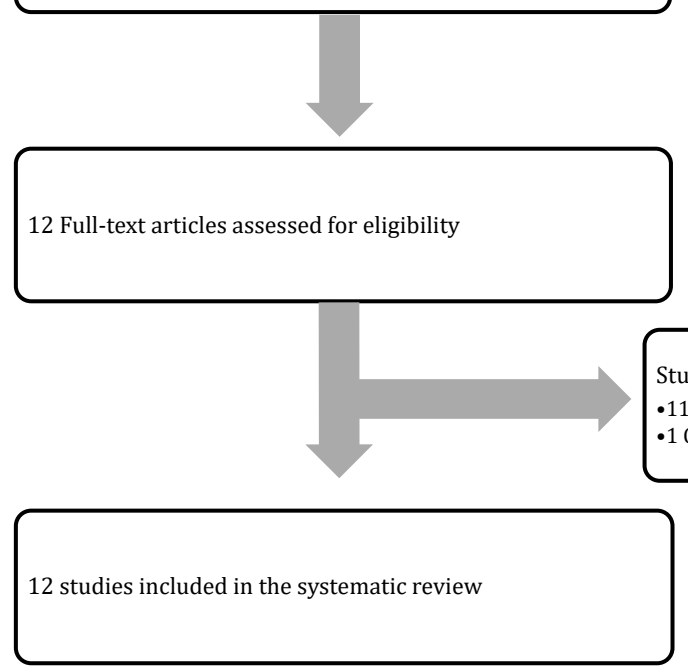

Figure 2. PRISMA Flow Diagram. This flowchart demonstrates how the cases of laser treatment for Darier Disease included in the systematic review were identified, screened, reviewed, included or excluded during selection. 


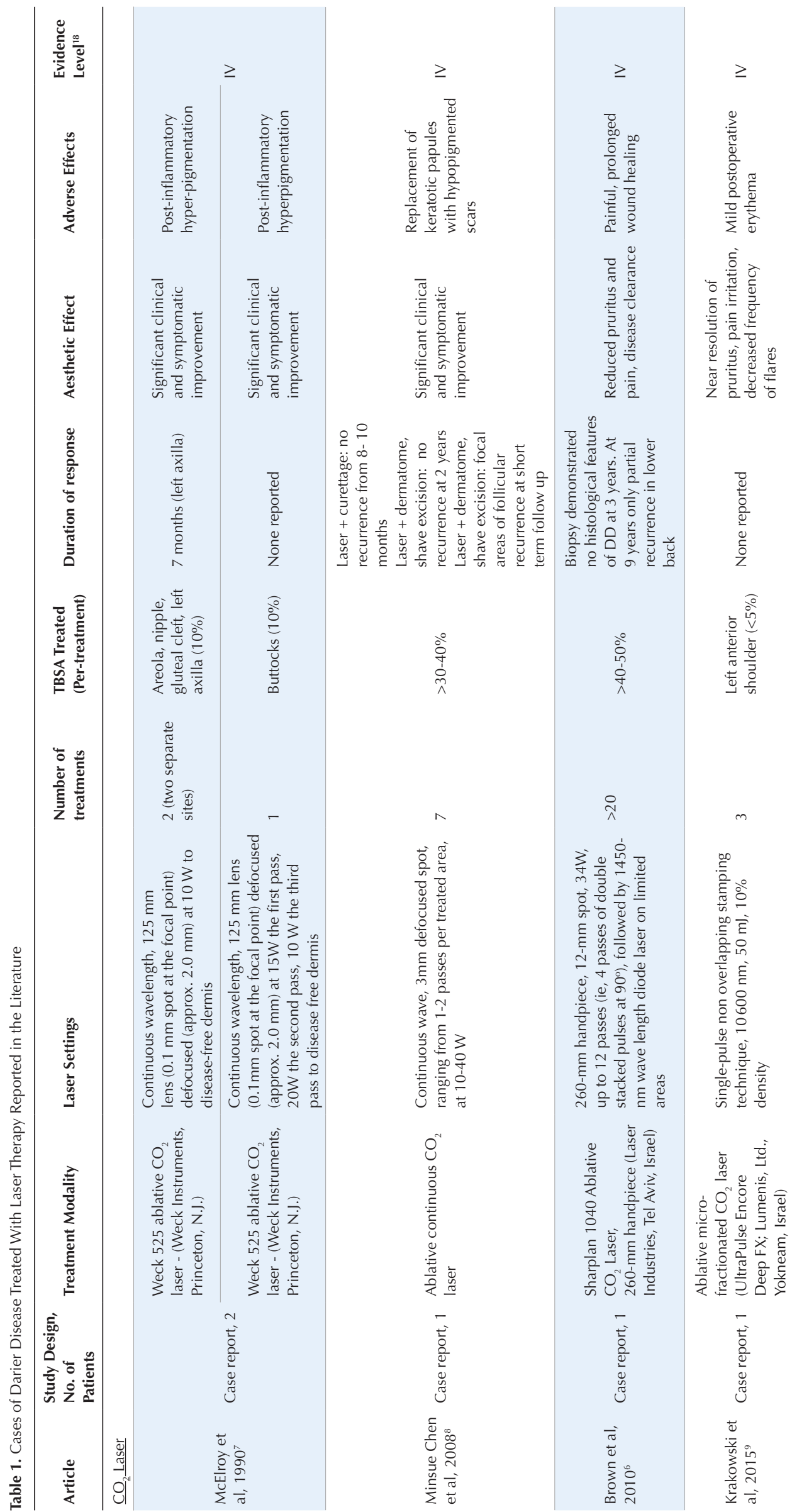




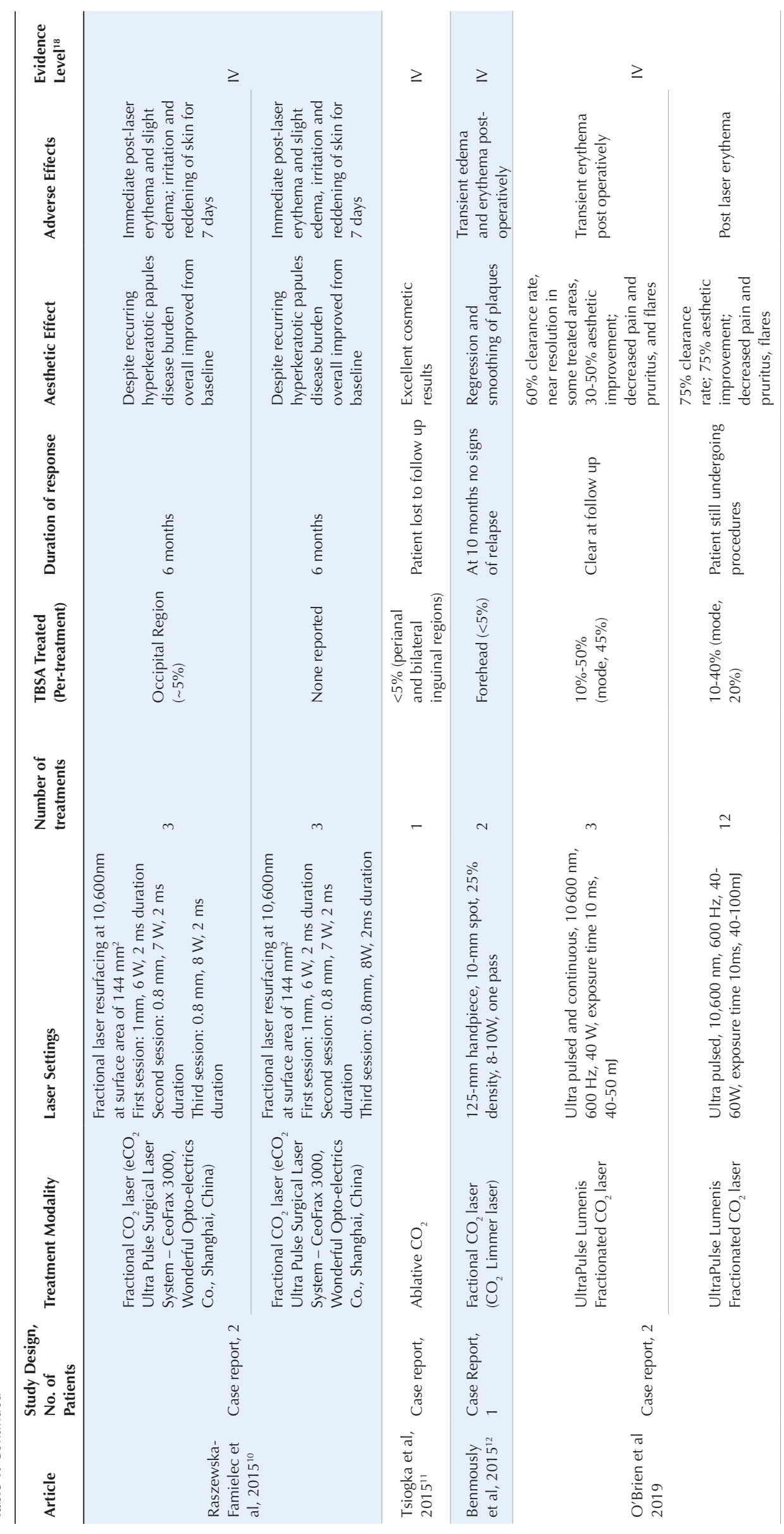




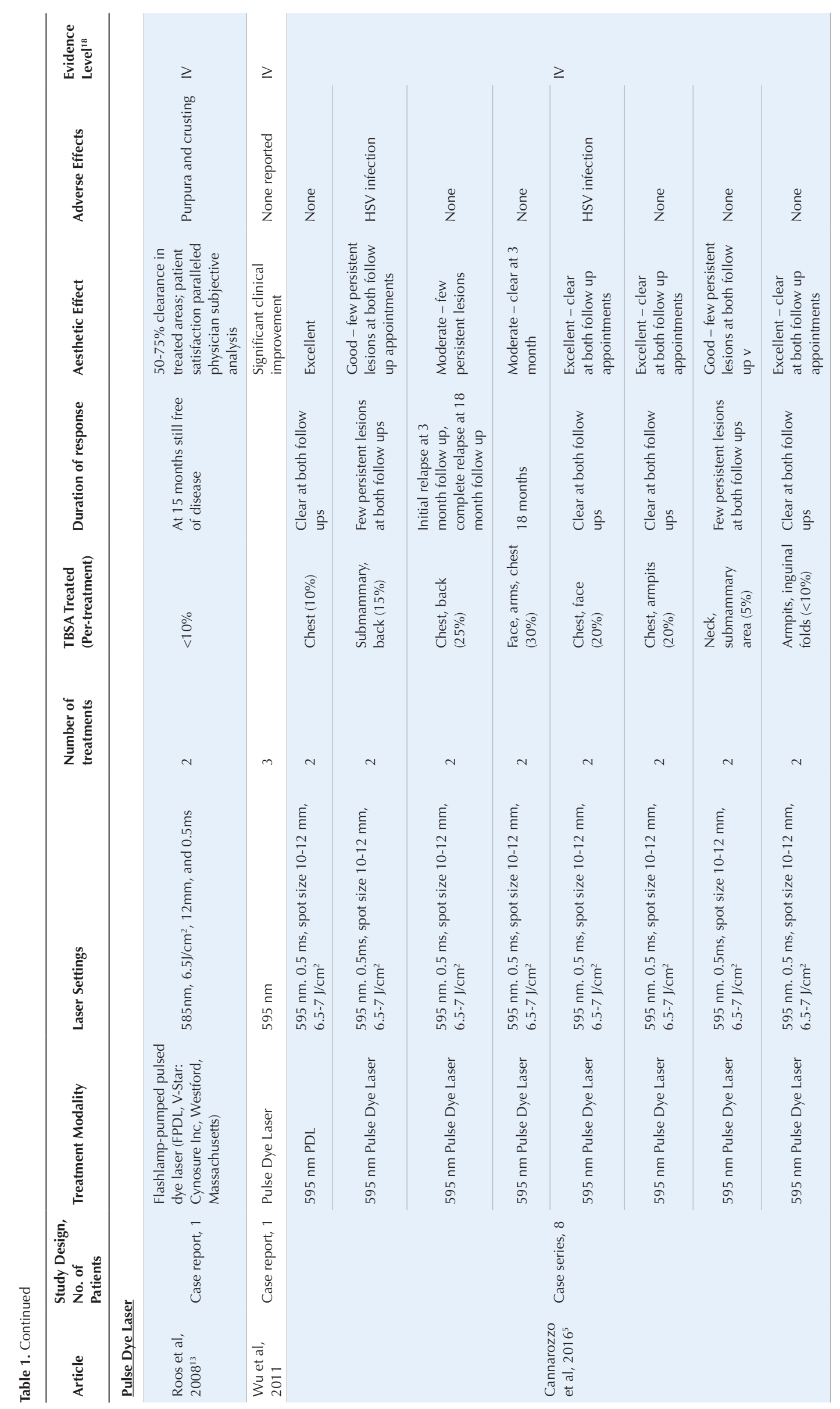




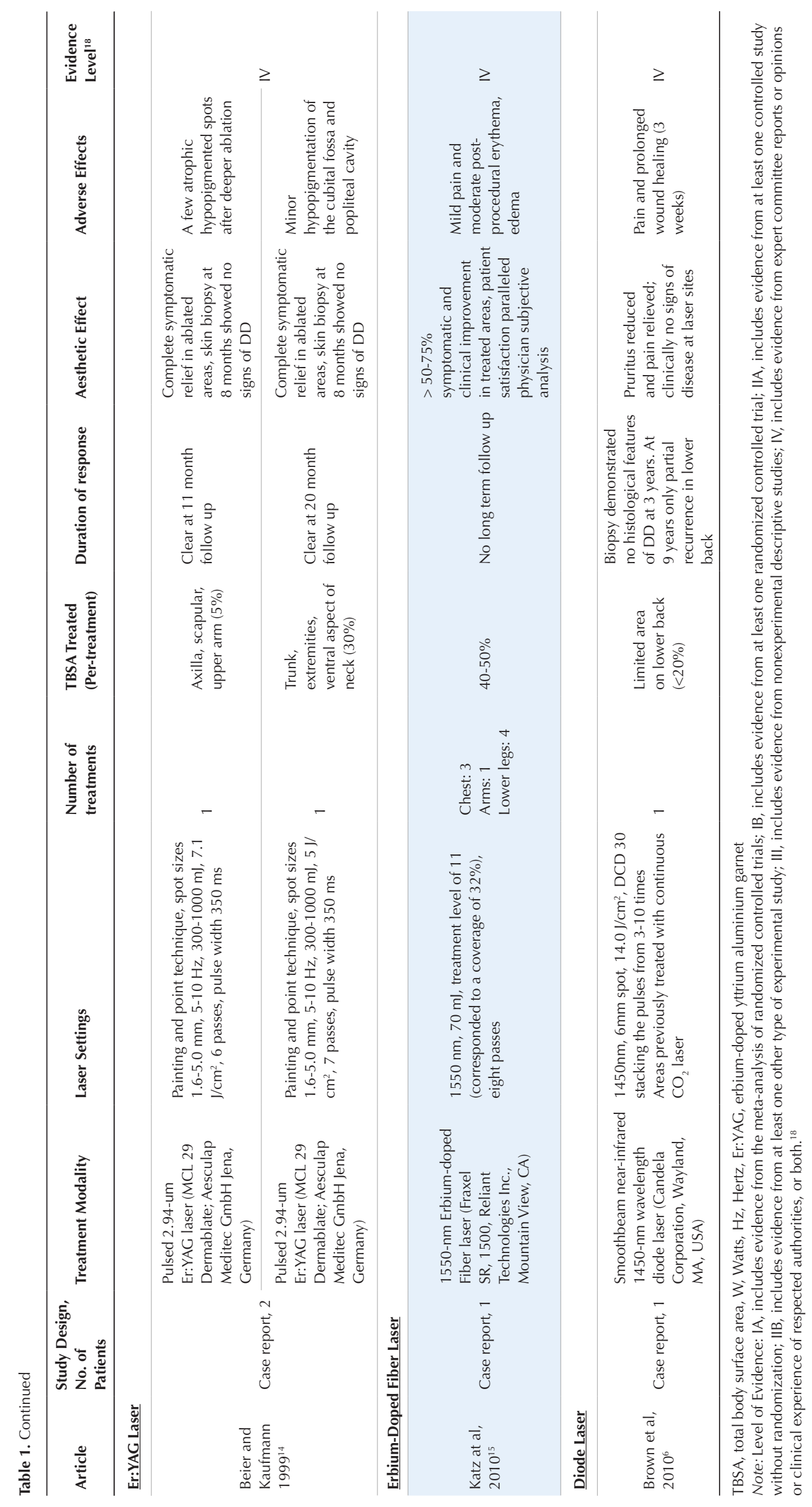


had greater than one procedure, with the average number of laser treatments per patient being $3.2 \pm 4.0$. Patients treated with Er:YAG underwent the fewest number of procedures, with both patients $(n=2)$ undergoing only one treatment session. In comparison, several patients treated with the $\mathrm{CO}_{2}$ laser underwent greater than 20 treatments. The interval time between the first and second treatments ranged from two weeks to nine months, with a two-month interval being the most frequently reported (mode 2 months, average $2.23 \pm 1.6$ months). Based on anatomical regions reported in each article, the TBSA treated per session was estimated by the author K.F.O. The TBSA treated ranged from 5-50\%, with an average TBSA of $19 \pm 14 \%$. There was an equal distribution of the TBSA treated among each laser type.

Follow-up after final laser treatment ranged from 0.5 to 20 months, with a variable degree of follow-up reported (standard deviation: 7 months). The time to response (average: $1.0 \pm 0.52$ months) was only reported in two-thirds of the cases reviewed, with improved clinical and symptomatic improvement being reported most commonly within four weeks following the first treatment. Of the reported cases in this review, both $\mathrm{CO}_{2}$ and PDL had the quickest time to response, with $\mathrm{CO}_{2}$ laser clinical response time ranging from immediate to 2.5 months. All patients undergoing PDL reported clinical or symptomatic improvement by 4 weeks. Of note, one of the required parameters from Cannarozzo et $\mathrm{al}^{5}$ was the time to response, which arguably skews the time to response in favor of the PDL. The duration of response was not longitudinally studied in the majority of cases reviewed. Final follow-up ranged from 1 month to 9 years. The single patient followed for 9 years underwent over 20 treatments, with both the $\mathrm{CO}_{2}$ laser and the Diode laser. This patient experienced partial recurrence at only one of the treated areas during the 9 years of follow-up. ${ }^{6}$ Two studies, one with Erb-YAG ${ }^{14}$ and the other with the $\mathrm{CO}_{2}$ laser, ${ }^{6}$ demonstrated biopsy-proven clearance of DD in areas treated with the laser at 18 and 24 months post final treatment respectively.

The outcome of treatment was determined by a range of subjective parameters from clinical improvement rated by the treating physician to clinical and/or symptomatic improvement rated by the patient. Subjective outcomes reported included pain, pruritus, weeping, and odor, and appearance was similar for all laser types. Adverse events differed by lasers. The most common adverse events from $\mathrm{CO}_{2}$ and Erb-YAG lasers were dyspigmentation, scarring, pain, and post-laser erythema and edema. Secondary infection with HSV infection was seen in two patients treated with PDL. ${ }^{5}$

\section{Discussion}

This is the first comprehensive review to synthesize the available results on laser therapy treatment in $\mathrm{DD}$. The exact mechanisms by which laser surgery improves DD remains unexplained, but postulations exist. Laser ablation of the papillary dermis is believed to be essential for success as more superficial treatments result in early relapse. ${ }^{10,16}$ The destruction of eccrine glands is also thought to reduce sweat-associated disease exacerbation. ${ }^{6}$ Finally, the side effect of scarring is postulated to decrease the constant external damage to the skin experienced by patients with DD. ${ }^{6}$ Taken together, the physical alterations in the treated skin change the threshold for the expression of the DD phenotype. ${ }^{6}$

At present, there are three major classes of dermatologic lasers used in DD: gas lasers, solid state lasers, and dye lasers, which come in ablative and nonablative forms. ${ }^{15}$

\section{Laser types used in Darier Disease Ablative \\ Carbon Dioxide (CO2) Laser}

This ablative laser comes in both fractionated and nonfractionated forms. ${ }^{17}$ The fractionated $\mathrm{CO}_{2}$ laser was one of the most commonly reported lasers used to treat DD (Table 1). The mechanism of action of the fractionated $\mathrm{CO}_{2}$ laser in DD is believed to be secondary to the destruction of the follicular and adnexal epithelium, which allows for remodeling and keratinocytes differentiation, increasing skin barrier thickness. ${ }^{8,12}$ The fractionated $\mathrm{CO}_{2}$ laser appears safe in the treatment of large BSAs, but as the percentage of the TBSA treated increases, there may be an associated risk of a DD flare.

Adverse events of the fractionated $\mathrm{CO}_{2}$ laser ranged from immediate postoperative erythema and pain to hyperpigmentation and downtime post procedure. Alternatively, skin softening and texture improvement have been noted immediately post-operatively.

Unless explicitly described as being fractionated, all previously reported $\mathrm{CO}_{2}$ laser treatments found in the literature review were assumed to be nonfractionated. ${ }^{6,7,8,11}$ Reported side effects from the nonfractionated $\mathrm{CO}_{2}$ laser cases reviewed were minimal and included hyperpigmentation and scarring ${ }^{7,8}$ side effects were also correlated with the increased number of treatments.

There has been one report of a patient who underwent shave excision prior to $\mathrm{CO}_{2}$ laser treatment with success. The preceding shave excision was associated with less subjective pain and faster healing time when compared to the procedures involving solely the $\mathrm{CO}$ laser. ${ }^{8} \mathrm{~A}$ different patient who left without papillary dermis following $\mathrm{CO}$ laser treatment also remained disease-free for up to $10^{2}$ years, supporting the fact that obliterating the culprit of disease, the dermis, leads to decreased numbers of flares. ${ }^{7}$

\section{Nonfractionated Er:YAG Laser}

The Er:YAG is a non-fractionated laser that emits light at 2940-nm wavelengths in the infrared range. Its 
unique absorption characteristics allow for selective deep follicular destruction which decreases sweating, another trigger of DD flares. ${ }^{14}$ Its advantage over a $\mathrm{CO}_{2}$ laser is its precise ablation technique, which occurred in in micrometer increments allowing for safe point-bypoint removal of keratotic papules and deeper thicker recalcitrant dermal lesions. ${ }^{14}$

The non-fractionated Er:YAG laser also appears to be efficacious in the treatment of DD. Both patients treated with the non-fractionated Er:YAG laser (the TBSA treated ranged from 5\%-30\%) in the literature required one treatment before going into complete remission. Additionally, one patient demonstrated biopsy negative DD in treated areas at 18 months after treatment. ${ }^{14} \mathrm{~A}$ significant decrease in itching was also reported after a single use of this laser. ${ }^{14}$ The side effects of the Er:YAG laser included post-operative edema and skin crusting after a single procedure. ${ }^{14}$ Dyspigmentation, with hypopigmentation being more common, was a common long-term side effect. ${ }^{14}$

\section{Nonablative \\ Erbium-Doped Fiber laser}

The 1550-nm erbium-doped fiber laser is able to penetrate depths of $382-1400 \mathrm{~mm}$, using energy levels up to $70 \mathrm{~mJ}$, while maintaining a high safety margin. ${ }^{15}$ In comparison to the ablative Er:YAG laser, a greater number of treatments and a more extensive TBSA treated per procedure have been shown to be safe. Additionally, in the case reviewed, the erbium-doped fiber laser demonstrated up to an $85 \%$ improvement of both clinical and subjective symptoms. ${ }^{15}$ Although this laser type may require more treatment sessions than an ablative laser, the overall complication rate was lower and recovery time appears to be shorter than the other laser types used in DD. ${ }^{15}$

\section{Diode Laser}

The 1450-nm diode laser is a light-emitting near-infrared laser. The mechanism of action is controlled tissue injury in the upper dermis while stimulating deep dermal remodeling and collagen production. ${ }^{16}$ Currently, there is only one reported case of a DD patient being treated with a diode laser, and that patient was additionally treated with the $\mathrm{CO}_{2}$ laser ${ }^{6}$; hence, efficacy and outcomes specifically related to the diode laser are unable to be determined. Recovery was reported to be painful and wound healing took three weeks. ${ }^{6}$ Unique outcomes in the patient treated with both the $\mathrm{CO}_{2}$ laser and the diode laser included lack of skin infections for several years and no histological features of residual DD on biopsy taken three years after treatment. ${ }^{6}$

\section{Pulsed Dye Laser}

A Pulsed Dye Laser is a 595nm laser used in the treatment of many inflammatory disorders. ${ }^{5}$ The mechanism of action is selective photothermolysis of oxyhemoglobin, capillary damage and edema of endothelial cells in dermal connective disuse, leading to the release of cytokines and growth factors from the dying cells. ${ }^{5,7} \mathrm{DD}$ is an inflammatory disease, and therefore the success of PDL is likely through its immunomodulatory properties. ${ }^{5} \mathrm{PDL}$ is considered safe and effective. In the single case series included in this review, ${ }^{5}$ a minimum of two produced favorable outcomes. The main side effect reported in PDL therapy was moderate pain and increased risk for HSV infection. ${ }^{5}$

\section{Conclusion}

This review demonstrates the clinical outcomes of five different dermatologic lasers in the treatment of DD. PDL and $\mathrm{CO}_{2}$ lasers, particularly fractionated $\mathrm{CO}_{2}$ lasers, were the most commonly reported lasers and demonstrated fast, long-lasting clinical and symptomatic improvements in Darier's patients. Ablative lasers, including both $\mathrm{CO}_{2}$ and Er:YAG, appear to be safe and effective in DD treatment despite the slightly higher side effect profile. With the addition of the fractional capability to the $\mathrm{CO}_{2}$ laser, the intensity of the ablative lasers is now better tolerated. It should be noted that the extent of the TBSA treated in patients should be carefully monitored, as we noted a risk of a DD flare with an increasing area of treatment in one of our cases. To our knowledge, we are the first to report this side effect of laser treatment of DD. Limitations of this review include a limited number of patient reports and lack of standardized long-term follow-up. Although more robust and controlled studies using ablative lasers are needed, laser treatment in $\mathrm{DD}$ appears to be a promising alternative therapy in DD.

\section{Ethical Considerations}

Not applicable.

\section{Conflict of Interests}

The authors declare no conflict of interest.

\section{Acknowledgement}

We are immensely grateful to Stephen J. Fernandez for his statistical expertise that greatly assisted with the analysis of this study.

\section{Supplementary Materials}

Supplementary file 1 contains Table S1.

\section{References}

1. Engin B, Kutlubay Z, Erkan E, Tüzün Y. Darier disease: A fold (intertriginous) dermatosis. Clin Dermatol. 2015;33(4):44851. doi: 10.1016/j.clindermatol.2015.04.009.

2. Savignac M, Edir A, Simon M, Hovnanian A. Darier disease: a disease model of impaired calcium homeostasis in the skin. Biochim Biophys Acta. 2011;1813(5):1111-7. doi: $\quad$ 10.1016/j.bbamcr.2010.12.006.

3. Bolognia JL, Schaffer JV, Cerroni L. Dermatology. 4th ed. 
Philadelphia: Elsevier Saunders; 2018.

4. Moher D, Liberati A, Tetzlaff J, Altman DG. Preferred reporting items for systemic reviews and meta-anlyses: the PRISMA statement. PLoS Med. 2009;6(7):e1000097. doi: 10.1371/journal.pmed.1000097.

5. Cannarozzo G, Bonciani D, Sannino M, Tamburi F, Morini C, Piccolo D, et al. Dye laser treatment for Darier disease: Results of a case series. Photomed Laser Surg. 2016;34(7):305-7. doi: 10.1089/pho.2015.4034.

6. Brown VL, Kelly SE, Burge SM, Walker NP. Extensive recalcitrant Darier disease successfully treated with laser ablation. Br J Dermatol. 2010;162(1):227-9. doi: 10.1111/j.1365-2133.2009.09540.x.

7. McElroy JA, Mehregan DA, Roenigk RK. Carbon dioxide laser vaporization of recalcitrant symptomatic plaques in Hailey-Hailey disease and Darier's disease. J Am Acad Dermatol. 1990;23(5 Pt 1):893-7. doi: 10.1016/01909622(90)70311-5.

8. Minsue Chen T, Wanitphakdeedecha R, Nguyen TH. Carbon dioxide laser ablation and adjunctive destruction for Darier-White disease (keratosis follicularis). Dermatol Surg. 2008;34(10):1431-4. doi: 10.1111/j.15244725.2008.34303.x.

9. Krakowski AC, Nguyen TA, Eichenfield LF. Treatment of segmental keratosis follicularis (Darier disease) Using Ablative Fractional Laser Resurfacing. Dermatol Surg. 2015;41(4):516-8. doi: 10.1097/DSS.0000000000000315.

10. Raszewska-Famielec M, Dudra-Jastrzebska M, Borzecki A, Chodorowskaf G. Darier-White disease treated with fractional CO2 laser in two cases. Dermatol Ther. 2015;28(4):254-7. doi: 10.1111/dth.12226.

11. Tsiogka A, Stückler C, Prodinger C, Koller J. Condyloma- like Darier's disease of the inguinal region: resolution after surgical excision and CO2 laser ablation. J Dtsch Dermatol Ges. 2015;13(11):1180-4. doi: 10.1111/ddg.12831.

12. Benmously R, Litaiem N, Hammami H, Badri T, Fenniche S. Significant alleviation of Darier's disease with fractional CO2 laser. J Cosmet Laser Ther. 2015;17(2):77-9. doi: 10.3109/14764172.2014.988728.

13. Roos S, Karsai S, Ockenfel HM, Raulin C. Successful treatment of Darier Disease with the flashlamp pumped pulse-dye laser. Arch Dermatol. 2008;144(8):1073-5. doi: 10.1001/archderm.144.8.1073.

14. Beier C, Kaufmann R. Efficacy of erbium:YAG laser ablation in Darier disease and Hailey-Hailey disease. Arch Dermatol. 1999;135(4):423-7.doi:10.1001/archderm.135.4.423.

15. Katz TM, Firoz BF, Goldberg LH, Friedman PM. Treatment of Darier's disease using a 1,550-nm erbium-doped fiber laser. Dermatol Surg. 2010;36(1):142-6. doi: 10.1111/j.15244725.2009.01369. $\mathrm{x}$

16. Preissig J, Hamilton K, Markus R. Current laser resurfacing technologies: A review that delves beneath the surface. Semin Plast Surg. 2012;26(3):109-16. doi: 10.1055/s-00321329413.

17. Gianfaldoni S, Tchernev G, Wollina U, et al. An overview of laser in dermatology: The past, the present and ... the Future(?). Open Access Maced J Med Sci. 2017;5(4):52630. doi: 10.3889/oamjms.2017.130.

18. Phillips B, Ball C, Sackett D Et al. Oxford Centre for Evidence-based Medicine-Levels of Evidence (March 2009). https://www.cebm.net/2009/06/oxford-centreevidence-based-medicine-levels-evidence-march-2009/. Accessed 25 August 2018 . 